\title{
Anthropometric and metabolic profile of children with gene polymorphisms for primary lactose intolerance
}

\author{
CORINA PIENAR ${ }^{1}$, LIVIU POP $^{1}$, MARILENA LĂZĂRESCU $^{2}$, \\ RADMILA COSTĂCHESCU ${ }^{1}$ and EDWARD ȘECLĂMAN ${ }^{3}$
}

\begin{abstract}
${ }^{1}$ Department of Pediatrics, 2nd Pediatrics Clinic, 'Victor Babes' University of Medicine and Pharmacy of Timisoara, 300041 Timisoara, Romania; ${ }^{2}$ Department of Pediatric Intensive Care, The Necker-Enfants Malades Hospital, University of Paris Descartes, 75015 Paris, France; ${ }^{3}$ Biochemistry Department, 'Victor Babes' University of Medicine and Pharmacy of Timisoara, 300041 Timisoara, Romania
\end{abstract}

Received May 28, 2021; Accepted June 29, 2021

DOI: $10.3892 /$ etm.2021.10768

\begin{abstract}
Primary lactose intolerance is caused by a genetically programmed loss in lactase production after 5-6 years of age. Milk and dairy products are often incriminated as a cause of gastrointestinal symptoms. Recent studies show that lactase persistence in adult life correlates with higher anthropometric indexes and an altered metabolic profile. We aimed to assess whether the presence of gene polymorphisms for primary lactose intolerance has an influence on the anthropometric and metabolic profile of children. We conducted a cross-sectional study, recruiting consecutive children evaluated at the 2nd Pediatric Clinic, Timisoara from May to August 2016. We enrolled 87 children aged 6-17 years [mean age $10.64 \pm 3.51$ years; $45(51.72 \%)$ girls]. Subjects were asked to complete an analogue visual scale of symptoms. We measured weight, height, blood pressure and calculated body mass index. The metabolic profile included fasting blood glucose, triglycerides and HDL cholesterol levels. We used strip genotyping to identify gene polymorphisms for primary lactose intolerance. According to the results, our study population was grouped into lactose tolerant $(n=42)$ and lactose intolerant $(n=45)$ groups. No differences were found in regards to weight, height, body mass index and blood pressure between the two study groups. Glucose, triglycerides and HDL cholesterol were similar in the lactose intolerant and lactose tolerant children. The presence
\end{abstract}

Correspondence to: Dr Corina Pienar, Department of Pediatrics, 2nd Pediatrics Clinic, 'Victor Babes' University of Medicine and Pharmacy of Timisoara, Piaţa Eftimie Murgu 2, 300041 Timisoara, Romania

E-mail: pienar.corina@umft.ro

Abbreviations: PLI, primary lactose intolerance; BMI, body mass index; SBP, systolic blood pressure; DBP, diastolic blood pressure; TG, triglycerides; HDLc, HDL cholesterol

Key words: lactose intolerance, polymorphisms, anthropometric profile, metabolic profile, children of gene polymorphisms for primary lactose intolerance did not influence the children's anthropometric and metabolic profile.

\section{Introduction}

Primary lactose intolerance (PLI), the most common form of disaccharide deficiency, is caused by a genetically programmed loss in lactase production after 5-6 years of age $(1,2)$. Lactase levels peak at birth following a descending trend afterward, until weaning age (2).

The lactase gene $(L C T)$ is located on the long arm of chromosome 2 and consists of 50 kilobases (kb) (3). PLI is considered the ancestral variant, while lactase persistence is an evolutionary advantage (4), consisting of 2 polymorphisms C/T13910 and G/A22018. These are upstream and downstream substitutions of 14 and $22 \mathrm{~kb}$ of the $5^{\prime}$ end of the lactase gene in a region that influences the lactase gene promoter (5). C/T13910 appears to be the dominant polymorphism, allele C being responsible for decreasing lactase expression $(3,5)$.

In adulthood, heterozygous individuals have moderately low lactase activity, and homozygous ( $C C, G G$, respectively) have undetectable levels of lactase at the surface of the intestinal mucosa. $T T$ or $A A$ genotypes correlate with lactose tolerance $(3,5)$.

Many individuals are carriers of the polymorphisms and retain their ability to digest lactose throughout their live. The beneficial effect of this mutation is still controversial, but it is thought to be the adaptation of our genome to cattle domestication and milk consumption. Consumption of dairy products has led to a rapid expansion of this mutation $(6,7)$. To the best of our knowledge, the PLI prevalence in Romania is unknown.

The most common symptoms of PLI in children include: Abdominal pain, abdominal distension, nausea, flatulence and diarrhea (8). Milk and dairy products are often incriminated as a cause of gastrointestinal symptoms, but their exclusion from the diet without a correct diagnosis may lead to nutritional imbalances, which may have a negative impact on weight and/or height $(5,9)$. However, recent studies show that lactase persistence in adult life correlates with higher anthropometric indexes and an altered metabolic profile (10-12). Even more, some authors propose that $L C T$ polymorphisms contribute to 
human obesity (10). Thus, the relationship between this nutrigenetic variant and obesity or metabolic syndrome have not yet been well defined.

The aim of the present study was to evaluate whether the presence of gene polymorphisms for PLI has an influence on the anthropometric and metabolic profile of children.

\section{Subjects and methods}

Study design. We conducted a cross-sectional study, recruiting consecutive children, evaluated in the 2 nd Pediatric Clinic, Timisoara, between May and August, 2016. A total of 87 children participated in the study after consent from the children or their legal guardians. At the time of inclusion all had to be between 6 and 18 years of age. Children on a diary-free diet, or who presented with malabsorption syndrome, diabetes mellitus, metabolic syndrome, obesity-related syndromes, or those receiving treatments that affect blood pressure, glucose and lipid metabolism were excluded from the study.

Ethical considerations. The study was conducted with full adherence to the international norms of medical ethics, as set out in the Helsinki Declaration. The study was approved by the 'Victor Babes' University of Medicine and Pharmacy Ethics Committee (approval no. 18/2016). Legal guardians and children above 16 years of age gave their consent for enrollment in the study.

Analyzed variables. In all children, the anthropometric profile was evaluated by measuring weight [kilograms $(\mathrm{kg})]$, height [meters (m)], systolic blood pressure (SBP, mmHg), diastolic blood pressure (DBP, $\mathrm{mmHg}$ ) and calculation of the body mass index [BMI, weight/square height $\left.\left(\mathrm{kg} / \mathrm{m}^{2}\right)\right]$.

All children and/or parents were asked to complete an analogue-visual scale for digestive symptoms associated with PLI (Data S1) (13). Children 6 and 7 years of age required parental assistance when completing the analogue-visual scale. Five children provided an incomplete scale, and thus were excluded from the symptom analysis. For the purpose of the analysis, symptoms from 0 to 2 were considered as no/mild symptoms, while symptoms between 4 and 10 were considered as moderate/severe.

A venous blood sample was collected and used to determine the metabolic profile of each child: Fasting glucose, triglycerides (TG) and HDL cholesterol (HDLc). The work-up was performed in the laboratory of 'Pius Brinzeu' County Emergency Hospital, Timisoara using spectrophotometry.

Molecular analysis. We used strip genotyping to identify children with genetic predisposition to PLI. This molecular diagnostic method is certified for in vitro diagnosis and permits a quick and reliable interpretation of the results. In addition, it only requires a drop of whole blood, which was collected and stored on a special support, GenoCard (Hain, Lifescience). Strip genotyping is a molecular diagnostic technique with high sensitivity and maximum specificity, which involves an amplification reaction and a reverse hybridization step. The strips are covered with samples complementary to the amplified nucleic acids.
As previously stated, within the $L C T$ gene, there are 2 polymorphisms responsible for lactase persistence/absence: The dominant $C / T 13910$ and $G / A 22018$. Homozygosity for the $C$ and $G$ allele are associated with PLI. Thus, children who were homozygous for at least one of the polymorphisms were considered lactose intolerant $(n=42)$, while the other were considered lactose tolerant $(n=45)$, irrelevant of their symptoms.

Definition of the profiles. For the purpose of our analysis, we defined an anthropometric profile which included weight, height, SBP and DBP, BMI. SBP and DBP measurements were compared with standards for age, sex and height.

We defined, as well, a metabolic profile which included: Fasting serum glucose, TG and HDLc. The cut-offs for the biologic markers were those proposed by our hospital's laboratory.

We sought subtle changes of these anthropometric and metabolic markers across gene polymorphism groups (lactose tolerant vs. lactose intolerant).

Statistical analysis. The results are expressed as means \pm standard deviation or frequencies for the continuous variables and for the ordinal variables, respectively. The t-test or Mann-Whitney test (based on the variable distribution) were employed to evaluate the differences in demographics, anthropometric and metabolic profiles between children with predisposition to PLI and those without this predisposition. All tests were performed for a confidence interval of 95\%, using the statistical package IBM SPSS Statistics 17 (IBM Corp.).

\section{Results}

Thirty children were homozygous for both polymorphisms, $C C 13910$ and GG22018, (34.5\%), while an additional 15 (17.2\%) were homozygous for one (Fig. 1). Our results were consistent with Hardy-Weinberg equilibrium.

Our study population consisted of 87 children: Aged 6-17 years [mean age 10.64 \pm 3.51 years; $45(51.72 \%)$ girls] (Table I). The study groups were homogenous when considering age and sex distribution (Table I).

Overall, moderate/severe symptoms were found in 20/87 children (Table I and Fig. 2. A total of 8/42 children with gene polymorphisms for PLI reported moderate/severe typical symptoms (Table I and Fig. 2). There was no difference in symptoms between children with gene polymorphisms for PLI and those without polymorphisms (Table I and Fig. 2).

Three of the 5 children that did not provide a complete symptoms' scale were lactose tolerant; the other 2 children had gene polymorphisms for PLI (lactose intolerant).

No significant differences were found in regards to the anthropometric profile when comparing children with vs. children without polymorphisms for PLI, in terms of weight, height, BMI, SBP and DBP (Table I).

As for the metabolic profile, fasting glucose, TG and HDLc levels were similar in children with and without gene polymorphisms for PLI (Table I).

\section{Discussion}

Approximately $65 \%$ of the human population has a reduced capacity to digest lactose after the weaning age. The prevalence 
Table I. Anthropometric and metabolic profile of children with and without gene polymorphisms for PLI (lactose tolerant).

\begin{tabular}{|c|c|c|c|}
\hline Features & Lactose tolerant $n=45$ & Lactose intolerant $n=42$ & P-value ${ }^{a}$ \\
\hline Mean age (years) & $11.09 \pm 3.59$ & $10.17 \pm 3.4$ & NS \\
\hline \multicolumn{4}{|l|}{ Sex, n $(\%)$} \\
\hline Girls & $21(46.66)$ & $24(57.14)$ & \multirow[t]{2}{*}{ NS } \\
\hline Boys & $24(53.34)$ & $18(42.86)$ & \\
\hline Weight (kg) & $42.92 \pm 16.94$ & $39.07 \pm 17.41$ & NS \\
\hline Height (cm) & $144.37 \pm 18.38$ & $148.67 \pm 18.06$ & NS \\
\hline BMI $\left(\mathrm{kg} / \mathrm{m}^{2}\right)$ & $19 \pm 5.08$ & $17.99 \pm 4.65$ & NS \\
\hline $\mathrm{SBP}(\mathrm{mmHg})$ & $99.8 \pm 5.49$ & $99.43 \pm 6.87$ & NS \\
\hline $\mathrm{DBP}(\mathrm{mmHg})$ & $67.74 \pm 10.47$ & $68.25 \pm 8.51$ & NS \\
\hline Glucose (mg/dl) & $85.47 \pm 13.5$ & $87 \pm 9.3$ & NS \\
\hline $\mathrm{TG}(\mathrm{mg} / \mathrm{dl})$ & $82.62 \pm 37.84$ & $76.74 \pm 35.59$ & NS \\
\hline HDLc (mg/dl) & $50.27 \pm 20.23$ & $44.1 \pm 12.04$ & NS \\
\hline \multicolumn{4}{|l|}{ Symptoms, n (\%) ${ }^{\mathrm{b}}$} \\
\hline No/Mild & $30(71.42)$ & $32(80)$ & \multirow[t]{2}{*}{ NS } \\
\hline Moderate/Severe & $12(28.58)$ & $8(20)$ & \\
\hline
\end{tabular}

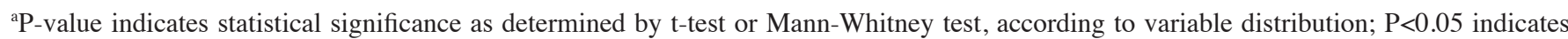

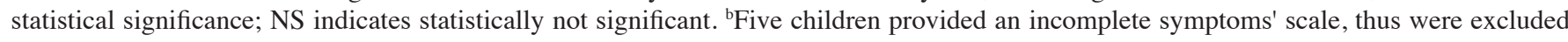
from the symptom analysis. PLI, primary lactose intolerance; BMI, body mass index; SBP, systolic blood pressure; DBP, diastolic blood pressure; TG, triglycerides; HDLc, HDL cholesterol.

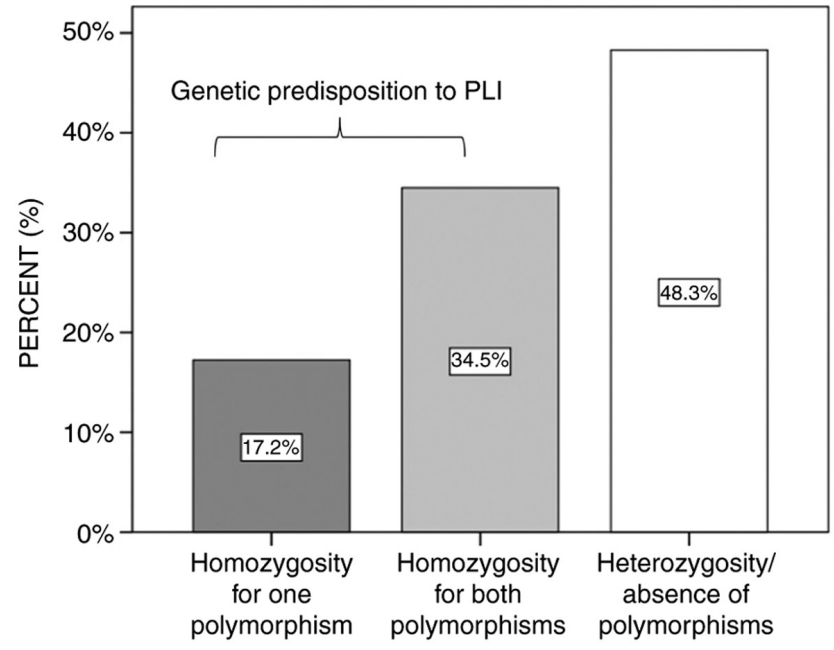

Figure 1. PLI-related genotypes in the study population. Irrelevant of their symptoms, children with homozygosity for one or both polymorphisms were considered lactose intolerant. The remaining children were lactose tolerant. PLI, primary lactose intolerance.

of primary lactose intolerance (PLI) is the lowest in populations with a long history of dependence on unfermented dairy products as an important source of food (14). Genetic mutations that determine lactase persistence appear to reflect the ancient history of milk consumption in different populations $(3,14)$.

When considering this finding, it is no surprise that PLI is most prevalent in individuals from East Asia, affecting more than $90 \%$ of adults in some of these communities (8). PLI is also extremely common in West African, Arabic and Jewish individuals (15). In Europe, the PLI prevalence has

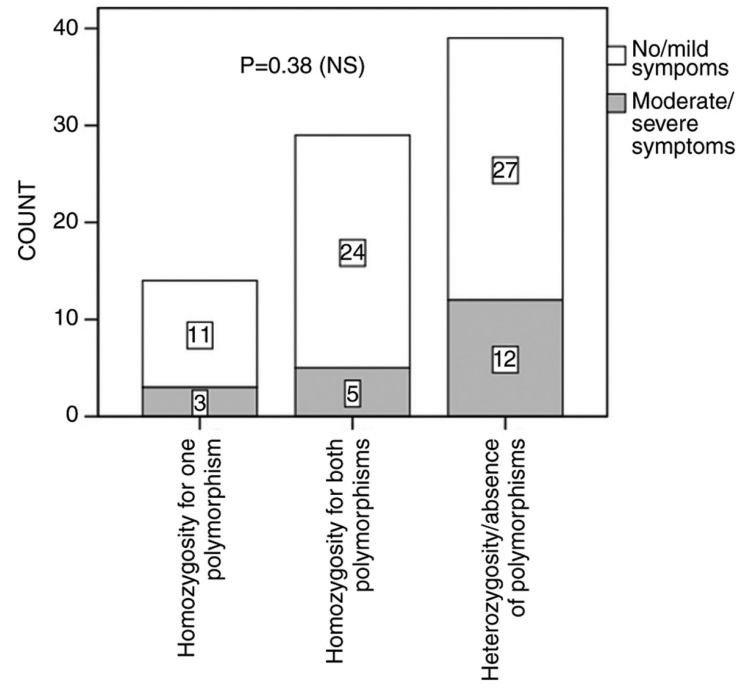

Figure 2. PLI-related symptoms across genotype groups: Children with and without gene polymorphisms for PLI. P=0.38; NS, statistically not significant as determined by the Mann-Whitney test. PLI, primary lactose intolerance.

a rising trend from north to south. Thus, only about $5 \%$ of Northern Europeans have PLI (3), in Central Europe about $30 \%$, while in Southern Europe PLI prevalence rises up to $70 \%$.

To the best of our knowledge, our study is the first to evaluate gene polymorphisms for PLI in the Romanian pediatric population. Our results show a PLI frequency of 51.7\%, which seems to follow European trends. Furthermore our results are in concordance with the Hardy-Weinberg equilibrium, making our results reliable. 
One must emphasize that only up to $50 \%$ of individuals with gene polymorphisms for PLI have typical symptoms (5). Many individuals with gene polymorphisms for PLI can consume milk and dairy products without symptoms $(1,3)$. Our study showed that only a minority of genetically predisposed children had typical symptoms. Furthermore, the symptom distribution was the same in both children with and without gene polymorphisms for PLI. Studies have shown that individual perception on the disease may influence greatly how individuals report symptoms $(16,17)$.

Our study did not show alterations in the anthropometric profile of the studied children. Weight, height and BMI were similar in children with and without gene polymorphisms for PLI. It is presumed that gastrointestinal symptoms associated with PLI lead to dietary dairy avoidance and to nutritional imbalances associated with lower anthropometric indexes (18). In our population a small number of children with genetic predisposition to PLI reported typical symptoms, thus avoidance behavior is unlikely. On the other hand, using larger cohorts Kettunen et al and Lamri et al showed that lactose tolerance in adulthood is associated with higher BMIs $(10,11)$. Furthermore, Kettunen et al also demonstrated that the BMI increase is mediated through increased weight and not decreased height (10). In a pediatric population of adolescents, Almon et al found that lactose tolerance leads to higher BMIs (12).

Our results suggest that a lactose tolerance-related increase in weight and BMI takes time and childhood may be the window of opportunity. Thus, we need to tackle these anthropometric changes through a balanced diet and individualized physical activity. Furthermore, a dairy-free diet recommendation, based on the presence of the gene polymorphisms for PLI must be discouraged.

Our study showed that the presence of gene polymorphisms for PLI did not influence the metabolic profile. Components of the metabolic syndrome definition, systolic blood pressure (SBP) and diastolic blood pressure (DBP), fasting glucose, triglycerides (TG) and HDLc levels, were similar in both study groups. Our results are consistent with the findings of Lehtimäki et al, who evaluated the metabolic profile of 2109 young Finns and showed that there was no significant difference between subjects with and without gene polymorphisms for PLI, in terms of fasting glucose, TG and HDLc levels (19). In contrast, Lamri et al, in their analysis of 3,575 French adults, showed that lactose tolerance and higher dietary lactose intake lead to higher risk for metabolic syndrome and type 2 diabetes (11). Still, fortunately, these pathological associations are reversible.

Our findings suggest that metabolic profile is not yet altered in childhood and once again emphasizes the need to promote a healthy life-style in the pediatric population.

To the best of our knowledge, this is the first study to analyze the presence of gene polymorphisms for PLI in a Romanian pediatric cohort. Although we included a small number of children, our results are consistent with the Hardy-Weinberg equilibrium and show a frequency of genetic predisposition to PLI in accordance to European trends. Still, due to the small sample and comparison with different populations, the results concerning the anthropometric and metabolic profile of children with genetic predisposition to PLI require careful interpretation. However, we need to emphasize the importance of a balanced diet, without unnecessary restrictions, throughout childhood and adolescence.

In conclusion, the presence of gene polymorphisms for PLI has no influence on the anthropometric and metabolic profile, irrespective of their symptoms.

\section{Acknowledgements}

Not applicable.

\section{Funding}

This work was supported by an internal grant of 'Victor Babes' University of Medicine and Pharmacy of Timisoara, Romania (PII-C4-TC-2016-08).

\section{Availability of data and materials}

The datasets used and/or analyzed during the current study are available from the corresponding author on reasonable request.

\section{Authors' contributions}

CP designed the study, analyzed and interpreted the patient data and drafted and revised the manuscript. LP made substantial contributions to the conception and design, interpreted the patient data and revised the manuscript critically for important intellectual content. ML was involved in data acquisition and interpretation, in drafting and revising the manuscript. RC was involved in data acquisition and interpretation, in drafting and revising the manuscript. ES performed the strip genotyping, analyzed and interpreted the patient data, contributed to conception and design and revised the manuscript critically. All authors read and approved the final manuscript for publication. CP, ML, RC, LP confirm the authenticity of all the raw data.

\section{Ethics approval and consent to participate}

The study was conducted with fully adherence to the international norms of medical ethics, as set out in the Helsinki Declaration. The study was approved by the 'Victor Babes' University of Medicine and Pharmacy Ethics Committee (approval no. 18/2016). Legal guardians and, where appropriate, children gave their consent for enrollment in the study.

\section{Patient consent for publication}

Not applicable.

\section{Competing interests}

The authors declare that they have no competing interests.

\section{References}

1. Suchy FJ, Brannon PM, Carpenter TO, Fernandez JR, Gilsanz V, Gould JB, Hall K, Hui SL, Lupton J, Mennella J, et al: National institutes of health consensus development conference: Lactose intolerance and health. Ann Intern Med 152: 792-796, 2010. 
2. Mattar R, Ferraz de Campos Mazo D and Carrilho FJ: Lactose intolerance: Diagnosis, genetic and clinical factors. Clin Exp Gastroenterol 5: 113-121,2012.

3. Zecca L, Mesonero JE, Stutz A, Poiree JC, Giudicelli J, Cursio R, Gloor SM and Semenza G: Intestinal lactase-phlorizin hydrolase (LPH): The two catalytic sites; the role of the pancreas in pro-LPH maturation. FEBS Lett 435: 225-228, 1998.

4. Järvelä I, Torniainen S and Kolho KL: Molecular genetics of human lactase deficiencies. Ann Med 41: 568-575, 2009.

5. Lomer MCE, Parkes GC and Sanderson JD: Review article: Lactose intolerance in clinical practice-myths and realities. Aliment Pharmacol Ther 27: 93-103, 2008.

6. Gerbault P, Roffet-Salque M, Evershed RP and Thomas MG: How long have adult humans been consuming milk? IUBMB Life 65: 983-990, 2013

7. Hubácek JA, Adámková V, Šedová L, Olišarová V, Adámek V and Tóthová V: Frequency of adult type-associated lactase persistence LCT-13910C/T genotypes in the Czech/Slav and Czech Roma/Gypsy populations. Genet Mol Biol 40: 450-452, 2017.

8. Hegar B and Widodo A: Lactose intolerance in Indonesian children. Asia Pac J Clin Nutr 24 (Suppl 1): S31-S40, 2015.

9. Heyman MB, Committee on Nutrition: Lactose intolerance in infants, children, and adolescents. Pediatrics 118: 1279-1286, 2006.

10. Kettunen J, Silander K, Saarela O, Amin N, Müller M, Timpson N, Surakka I, Ripatti S, Laitinen J, Hartikainen AL, et al: European lactase persistence genotype shows evidence of association with increase in body mass index. Hum Mol Genet 19: 1129-1136, 2010.

11. Lamri A, Poli A, Emery N, Bellili N, Velho G, Lantieri O, Balkau B, Marre M and Fumeron F: The lactase persistence genotype is associated with body mass index and dairy consumption in the D.E.S.I.R. study. Metab Clin Exp 62: 1323-1329, 2013.
12. Almon R, Nilsson TK, Sjöström M and Engfeldt P: Lactase persistence and milk consumption are associated with body height in Swedish preadolescents and adolescents. Food Nutr Res: 55, 2011 doi: 10.3402/fnr.v55i0.7253.

13. Tomba C, Baldassarri A, Coletta M, Cesana BM and Basilisco G: Is the subjective perception of lactose intolerance influenced by the psychological profile? Aliment Pharmacol Ther 36: 660-669, 2012.

14. Gerbault P: The onset of lactase persistence in Europe. Hum Hered 76: 154-161, 2013

15. Fang L, Ahn JK, Wodziak D and Sibley E: The human lactase persistence-associated SNP-13910*T enables in vivo functional persistence of lactase promoter reported transgene expression. Hum Genet 131: 1153-1159, 2012

16. Casellas F, Aparici A, Pérez MJ and Rodríguez P: Perception of lactose intolerance impairs health-related quality of life. Eur J Clin Nutr 70: 1068-1072, 2016.

17. Kull M, Kallikorm R and Lember M: Impact of molecularly defined hypolactasia, self-perceived milk intolerance and milk consumption on bone mineral density in a population sample in Northern Europe. Scand J Gastroenterol 44: 415-421, 2009.

18. Rozenberg S, Body JJ, Bruyere O, Bergmann P, Brandi ML, Cooper C, Devogelaer JP, Gielen E, Goemaere S, Kaufman JM, et al: Effects of dairy products consumption on health: Benefits and beliefs-a commentary from the Belgian Bone Club and the European Society for Clinical and Economic Aspects of Osteoporosis, Osteoarthritis and Musculoskeletal Diseases. Calcif Tissue Int 98: 1-17, 2016.

19. Lehtimäki T, Hutri-Kähönen $N$, Kähönen $M$, Hemminki J, Mikkilä V, Laaksonen M, Räsänen L, Mononen N, Juonala M, Marniemi J, et al: Adult-type hypolactasia is not a predisposing factor for the early functional and structural changes of atherosclerosis: The Cardiovascular Risk in Young Finns Study. Clin Sci (Lond) 115: 265-271, 2008. 